\title{
Novel nitrogen-containing heterocyclic compounds in GPR109A as an anti-hyperlipidemic: Homology modeling, docking, dynamic simulation studies
}

\author{
Prajakta B. KOTHAWADE*1D, Kiran Bharat LOKHANDE*2 (D), Kakumani Venkateswara SWAMY 2 (D), \\ Sohan S. CHITLANGE ${ }^{1}$ (D), Asha B. THOMAS $* * 1$ (D) \\ 1 Department of Pharmaceutical Chemistry, Dr. D. Y. Patil Institute of Pharmaceutical Science and Research, attached \\ to SPPU, Pimpri, Pune, Maharashtra, India - 411018. \\ 2 Bioinformatics Research Laboratory, Dr. D. Y. Patil Biotechnology and Bioinformatics Institute, Dr. D. Y. Patil \\ Vidyapeeth, Pune, Maharashtra, India - 411033. \\ ** Corresponding Author. E-mail: asha.thomas@dypvp.edu.in (A.T.) Tel +091-988-123 6220. \\ * These authors contributed equally.
}

Received: 10 January 2020 / Revised: 07 April 2020/ Accepted: 13 April 2020

\begin{abstract}
Niacin or nicotinic acid therapy leads to reduction of the level of Low-density Lipoprotein cholesterol (20-40\%) with significant elevation of High-Density Lipopreoin cholesterol level (20-35\%). From research, it was said that Nicotinic acid might exert its positive action by activating the G-protein-coupled receptor (GPCR) which is found on adipocytes. GPR109A (family of GPCR) receptor was important for nicotinic acid (niacin) for its anti-lipolytic effects. As GPR109A is a targeted receptor for the treatment of dyslipidemia, its structural analysis needs to be elucidated. But the Protein 3D structure of target was not available at Protein Data Bank (PDB), so we have generated its structure through homology modeling and validation was carried out. Screening of top lead molecules with the help of various computational approaches like molecular-docking and molecular dynamic (MD) simulations studies with different online tools were carried out. The docking results showed that the lead compound 2B [(R)-methyl 2-(2(1H-indol-3-yl) acetamido)-3-(1H-indol-3-yl) propionate] revealed significant binding energy value $(-30.54 \mathrm{kcal} / \mathrm{mol})$ as that with the nicotinic acid which is a standard drug $(-17.68 \mathrm{kcal} / \mathrm{mol})$. In addition to that, Molecular-Dynamic (MD) simulations analysis proved that compound $2 \mathrm{~B}$ has lesser variations throughout the simulation period as represented by the root-mean-square deviation (RMSD) and root-mean-square fluctuation (RMSF) graphs. Current in silico study describes the modeling of novel heterocyclic compounds as antihyperlipidemic drugs for the treatment of dyslipidemia. This study also describes a deeper idea about the structural information of the lead compound $2 \mathrm{~B}$ and its entire molecular interactions against GPCR109A and provides a hypothetical guideline to utilize this compound as an antihyperlipidemic for the treatment of dyslipidemia.
\end{abstract}

KEYWORDS: G-protein coupled receptor (GPCR); homology modeling; antihyperlipidemic drugs; nicotinic acid; molecular docking and molecular dynamic simulations.

\section{INTRODUCTION}

Nicotinic acid (Niacin), the water-soluble vitamin helps to reduce plasma lipid levels of total cholesterol (TC), free fatty acids (FFA), triglycerides (TG) when administered to humans beings [1, 2]. Nicotinic acid robustly increases high-density lipoprotein levels compared to other anti-hyperlipidemic drugs [3]. How nicotinic acid acts by lowering lipid levels in the body, this metabolism is still not clear. Harmful side effects shown by nicotinic acid such as flushing (facial reddening), reduced glucose tolerance or gastral intestinal effects decrease patient compliance [4]. Nicotinic acid plays an important role by inhibiting fat cell lipolysis by the activation of a G protein-coupled receptor (GPCR) and successive inhibition of cAMP configuration [5, 6] and [7]. In 2003, scientists identified three G Protein-coupled receptors (GPR109A, GPR81, and GPR109B) that binds to nicotinic acid with projected similarity [8-10]. The GPR109A receptor, couples to $\mathrm{G}$ protein of Gi family, which is expressed mainly in adipocytes and immune cells. The receptors GPR109A and GPR81 both exist in humans as well as in rodent species [11]. The antihyperlipidemic effects of nicotinic acid cause a reduction in FFA and TG, but in mice lacking PUMA-G, anti-

How to cite this article: Kothawade P, Lokhande K, Thomas A, Swamy K, Chitlange S, Novel nitrogen-containing heterocyclic compounds in GPR109A as an anti-hyperlipidemic: Homology modeling, docking, dynamic simulation studies,. J Res Pharm. 2020; 24(4): 452-463. 
lipolytic effects are not observed [9]. Thus, it was proved that GPR109A is the receptor, which brings about the anti-lipolytic effects of nicotinic acid.

In earlier studies, Tanura et al. generated a structural homology model for receptor GPR109 A/ B, they used the X-ray structure of Rhodopsin [12,13] from protein data bank (PDB id: 1HZX) [14] as a template. The validation of the 3D model of the GPR109A receptor was done by checking the quality as well as the stability of the model. The model was validated by analyzing the stereochemical quality of protein structure by PROCHECK [15] and the stability was confirmed by molecular dynamics (MD) run (root mean square deviation (RMSD), $1.7 \AA$ ). In GPCR for small ligands, the binding site was not formed by transmembrane helices (TMH) 3, 5 and 6 but the binding site was formed by transmembrane helices 2, 3 and 7[13]. The acidic group of nicotinic acid interacts with the basic amino acid Arg111 at TMH3, whereas the pyridine ring of nicotinic acid is fixed between Trp91 at the junction TMH2/ECL1, Phe276, and Tyr284 at TMH7. The pyridine nitrogen is also bound to Ser178 at ECL2 via H bond. Asn86 (TMH2) restrains the orientation of Trp91 by hydrogen bond, and Phe180 (ECL2) restrains this orientation of Phe276 by aromatic interactions leading to an appropriate and inflexible binding cleft.

Through the Gi -protein-mediated inhibition of adenylyl cyclase; the nicotinic acid receptor is activated in adipocytes, which results in the reduction of TAG to FFAs. The anti-lipolytic effects of nicotinic acid that are essential for reducing FFA levels and subsequent decrease in TAG plasma levels are canceled, in mice lacking PUMA-G [9]. Mainly nicotinic acid (NA) increases HDL levels but it is still not clearly understood how nicotinic acid increases HDL. From several studies, it was clear that nicotinic acid decreases the catabolism of Apo A-I-containing lipoprotein particles [16, 3]. The main side effect of nicotinic acid flushing, which was observed at very small doses (50-100mg per os) of nicotinic acid and reddening occurs mainly in the upper body, neck, and face [17]. The NSAID'S can reduce initial flushing whereas they do not alter the effects of nicotinic acid on lipid levels. From recent studies, it is clear that the cutaneous flushing effect is mediated by GPR109A. Thus in PUMA-G deficient mice nicotinic acid does not induce flushing, although this response can be restored by transplanting wild type bone marrow onto irradiated GPR109A deficient animals [18].

In this article, we have designed a new GPR109A receptor by homology modeling approach and different agonists of the GPR109A receptor. Nicotinic acid and its 80 derivatives were docked with modeled GPR109A receptor, by using FlexX software, which is used for batch docking. The docking result shows that binding energy for all the compounds was less than nicotinic acid, which is a natural agonist. The best 6 compounds were selected for further docking analysis.

\section{RESULTS AND DISCUSSION}

\subsection{Model validation}

The model generated by SWISS-MODEL [19] for GPR109A was subjected to validation using PROCHECK [20], PROSA Web server [21] and SAVES [22]. The template and model alignment was done and the alignment score was 0.012 . The root means square deviation (RMSD) was $0.545 \AA$ (Figure 1). Protein and ligands were submitted to PDBsum [23] and Ramachandran plots were generated. The model was validated based on PDBsum generated Ramachandran Plot statistics (Table 1). Figure 2 shows that the generated model, the one with the maximum number of residues in the allowed region (yellow) and the lowest number of residues in the disallowed region (Red) was considered as the appropriate model for GPR109A, for further analysis. The PDBsum generated Ramachandran plot for the selected model showed that number of residues present in favored region $245(90.7 \%)$, number of residues present in allowed region $20(7.4 \%)$ and number of Residues in generously allowed regions $3(1.1 \%)$ and Residues in disallowed regions $02(0.7 \%)$. Residues in disallowed regions are Phe25 (A) and Pro302 (A).

The Ramachandran plot shows the phi-psi torsion angles for all residues in the structure (except those at the chain termini). Glycine residues are separately identified by triangles as these are not restricted to the regions of the plot appropriate to the other side chain types. The shading on the plot represents the different regions as shown in Figure 2, the darkest areas in red correspond to the "core" regions showing the most favorable combinations of phi-psi values. Ideally, one would hope to have over $90 \%$ of the residues in these "core" regions. The percentage of residues in the "core" regions is one of the better guides to stereochemical quality. PROSA analysis of the selected model revealed that the model has structures resembling native structures with a Z -score of -3.95. Whereas for SAVES-VCLA-DOE LAB with overall quality factor 91.87. This confirms that the model generated by the SWISS model is highly reliable and can be considered for further studies. 


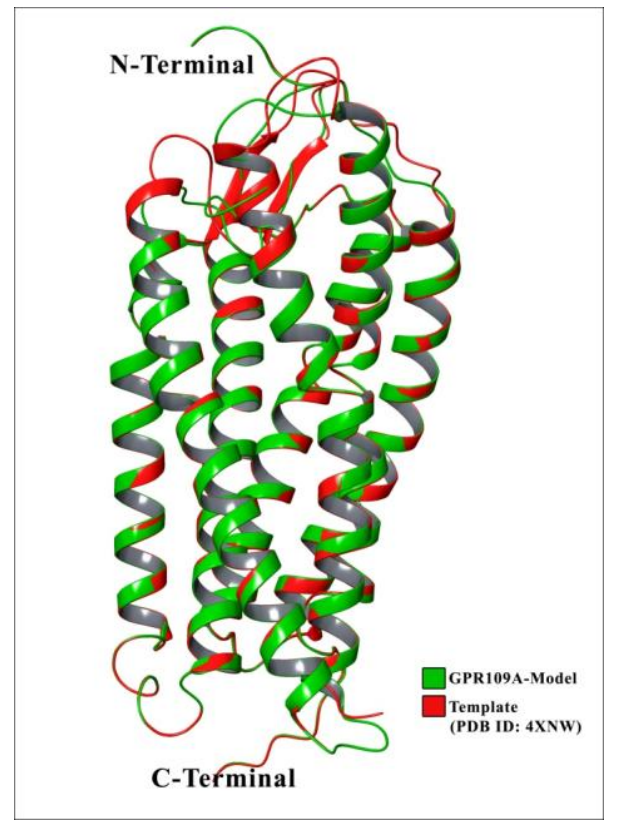

Figure 1. Model Template Alignment: (Superimposition of GPR109A with the template (PDB id 4XNW) by maestro and RMSD is $0.545 \AA$. The template structure is shownin Red and target with Green colour. $0.012 \%$ of amino acids deviated with the templatestructure.

Table 1. Ramachandran plot statistics.

\begin{tabular}{lll}
\hline Residual Properties & $\begin{array}{l}\text { Number of } \\
\text { Residues }\end{array}$ & $\begin{array}{l}\text { Total\% of } \\
\text { Residues }\end{array}$ \\
\hline Residues in most favoured regions [A,B,L] & 245 & 90.7 \\
Residues in additional allowed regions [a,b,l,p] & 20 & 7.4 \\
Residues in generously allowed regions [ $\mathrm{a}, \mathrm{b}, \mathrm{l}, \mathrm{p}]$ & 3 & 1.1 \\
Residues in disallowed regions & 2 & 0.7 \\
Number of end-residues (excl. Gly and Pro) & 3 & 1.1 \\
Number of glycine residues (shown as triangles) & 12 & 4.4 \\
Number of proline residues & 11 & 4.0 \\
Number of non-glycine and non-proline residues & 270 & 100.0 \\
\hline
\end{tabular}

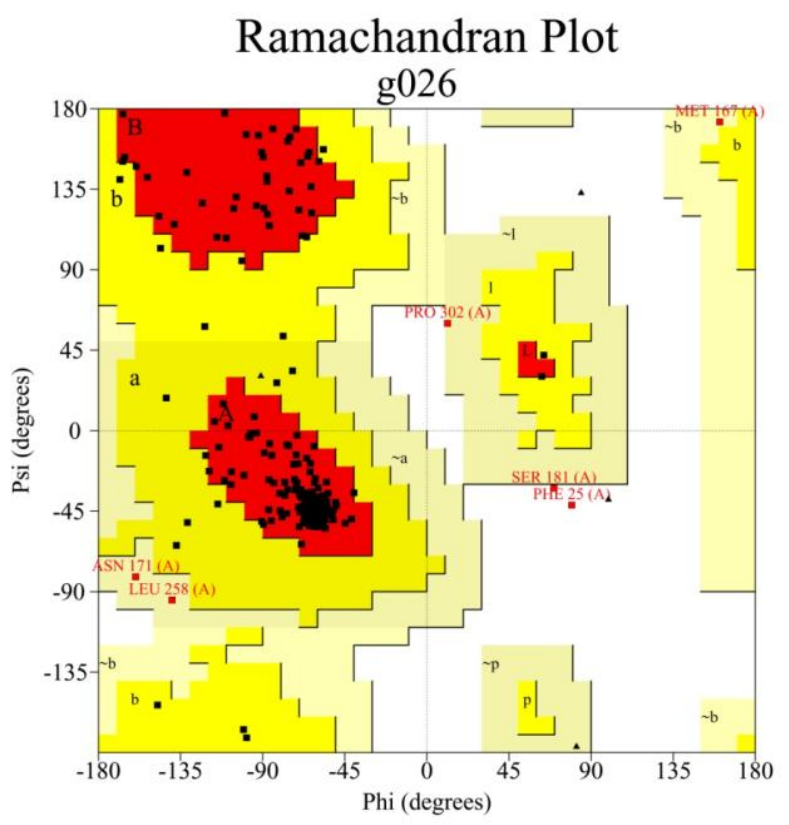

Figure 2. Model validation by Phi-Psi plot showing that the Phi-Psi plot cluster of the model is of high quality with a minimal outlier. (Red: Favored Region, Yellow: Allowed Region) 


\subsection{Secondary structure analysis}

The secondary structure characterization of the GPR109A model was done through GPCRdb [24] which provides information on the number of helices and loops. Figure 3(a) shows the secondary structure of GPR109A, comprises of 7 helices, 3 intracellular loops, 3 extracellular loops, N-terminal and C-terminal. GPR109A color model (Figure 3(a)) N-Terminal starts at Met1 to Asp24 (Red), TM1 contains amino acids from Phe25-His55 (Green), TM2 contains Lys60-Arg90 amino acids (Blue), TM3 contains Gly96-His131 amino acids (violet), TM4 contains Ser140 to Lys166 (Cyan) amino acids, TM5 contains Phe180 - Gln217 (yellow) amino acids, TM6 contains Lys225-Thr260 (Grey) amino acids, and TM7 contains Asn265-Ser297 (Pink dark Salmon) amino acids. H8 lysozyme was removed, ICL1 (Leu56-Trp59), ICL2 (Pro132-Leu139), ICL3 (Aer218-Ala224) (magenta), ECL1 (Trp91-Phe95), ECL2 (Met167-Ser179), ECL3 (Ser261-Gln264) (Red Orange), whereas C-terminal contains Ser298-Leu308 (Orange) amino acids. The top view of the GPR109A receptor model is shown in Figure 3(b).
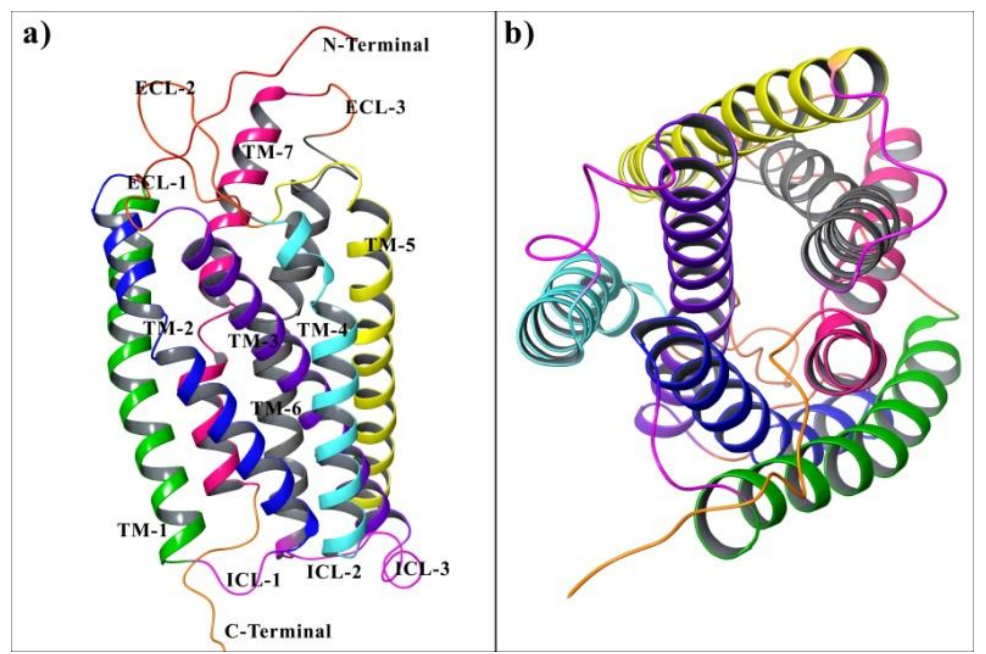

Figure 3. a) GPR109A modeled receptor shown in a different color with transmembrane helices. (b) Top view of GPR109A modeled receptor.

\subsection{Active site analysis}

Once the final model was built and validated for GPR109A the possible binding sites were identified. From the PDBsum entries [24] and literature review [13] of the templates chosen for GPR109A, their binding sites were analyzed. Figure 4(a) shows active site residues that are Ser181, Ser178, Phe180, Ser179, Phe276, Leu176, Trp91, Phe21, Val272, His259, Asp273, Tyr269, Ile254, and Thr260. Figure 4(b) shows the Snake diagram with interacting residues in transmembrane helices.

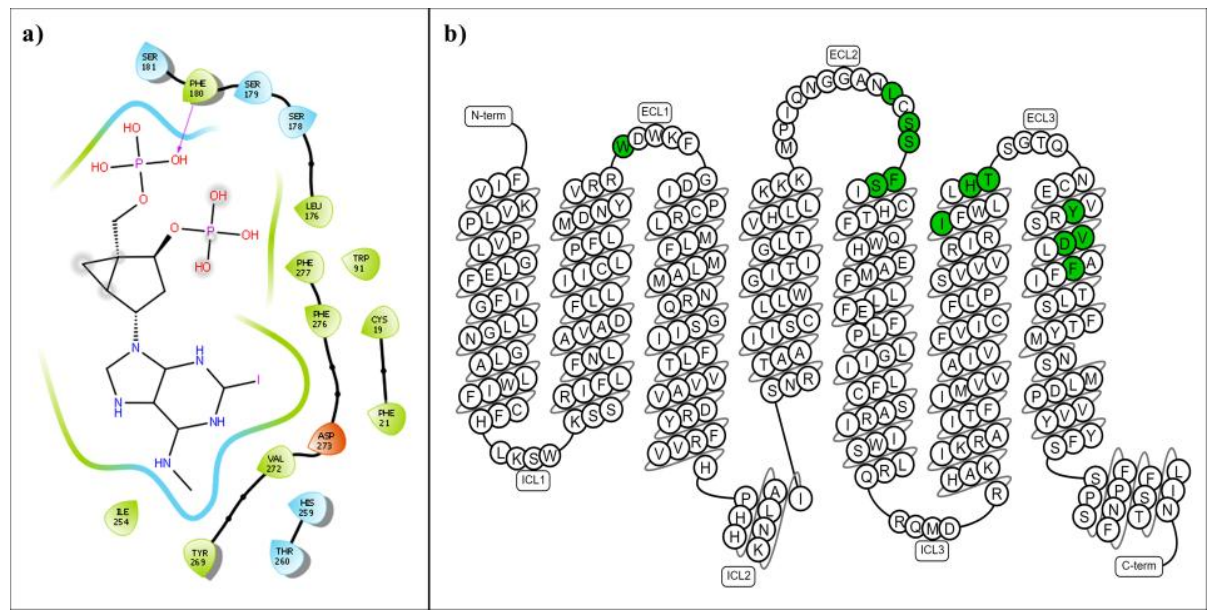

Figure 4 a) 2-D diagram shows residues involved in the binding pocket of the GPR109A model receptor. (b) Snake diagram showing different helixes with interacting residues shown in green color. 


\subsection{Docking analysis}

2D and 3D structures are visualized in Maestro software [25]. All the ligands were energy minimized and partial charges were added on OPLS-2005 software [26]. To understand the binding mode, nicotinic acid and its 80 derivatives were docked with GPR109A, by using FlexX software [27] which is used for batch docking. The docking result shows that binding energy for all the compounds was less than nicotinic acid which is a natural agonist. The best 6 compounds were selected for further docking analysis. The binding energy of the best compound with nicotinic acid is shown in Table 2 and their 2D structures are shown in Table 3.

Table 2. Docking analysis of nicotinic acid and screened compounds with GPCR109A.

\begin{tabular}{|c|c|c|c|c|}
\hline $\begin{array}{l}\text { Compounds } \\
\text { Name }\end{array}$ & $\begin{array}{l}\text { Binding Energy } \\
\text { (kcal/mol) }\end{array}$ & $\begin{array}{l}\text { Interacting } \\
\text { Residues }\end{array}$ & Bond Type & $\begin{array}{l}\text { Bond Distance } \\
(\AA)\end{array}$ \\
\hline \multirow{5}{*}{ Nicotinic acid } & \multirow{5}{*}{-17.68} & Ile254 & Aromatic HB & 2.5 \\
\hline & & Phe180 & H-bond & 1.83 \\
\hline & & Phe180 & $\Pi-\Pi$ stacking & 4.32 \\
\hline & & Trp256 & H-bond & 1.89 \\
\hline & & Trp256 & П-п stacking & 5.08 \\
\hline \multirow{7}{*}{$2 B$} & \multirow{7}{*}{-30.5487} & Trp91 & $\Pi-\Pi$ stacking & 5.08 \\
\hline & & Trp91 & $\Pi-\Pi$ stacking & 5.08 \\
\hline & & Phe180 & $\Pi-\Pi$ stacking & 5.15 \\
\hline & & Phe180 & H-bond & 2.08 \\
\hline & & Ser181 & H-bond & 2.18 \\
\hline & & Asp273 & Aromatic HB & 1.87 \\
\hline & & Phe276 & $\Pi-\Pi$ stacking & 4.73 \\
\hline \multirow{5}{*}{$5 \mathrm{~J}$} & \multirow{5}{*}{-27.1781} & Trp91 & $\Pi-\Pi$ stacking & 4.71 \\
\hline & & Ser178 & H-bond(side chain) & 1.59 \\
\hline & & Ser179 & H-bond & 1.95 \\
\hline & & Ser181 & H-bond & 2.16 \\
\hline & & Phe180 & H-bond & 2.08 \\
\hline \multirow{5}{*}{$3 B$} & \multirow{5}{*}{-26.7115} & Phe21 & П-п stacking & 5.30 \\
\hline & & Phe21 & $\Pi-\Pi$ stacking & 5.30 \\
\hline & & Tyr87 & H-bond(side chain) & 2.49 \\
\hline & & Ser179 & H-bond & 2.05 \\
\hline & & Phe180 & П-п stacking & 5.31 \\
\hline \multirow{6}{*}{$4 \mathrm{~B}$} & \multirow{6}{*}{-26.3793} & Ser179 & H-bond(side chain & 1.99 \\
\hline & & Phe180 & H-bond & 2.44 \\
\hline & & Ser181 & H-bond & 1.68 \\
\hline & & Trp256 & H-bond(side chain & 2.11 \\
\hline & & Trp256 & $\Pi-\Pi$ stacking & 5.2 \\
\hline & & Trp256 & $\Pi-\Pi$ stacking & 5.43 \\
\hline \multirow{6}{*}{$5 \mathrm{E}$} & \multirow{6}{*}{-26.32} & Ser178 & H-bond & 1.55 \\
\hline & & Ser179 & H-bond & 1.97 \\
\hline & & Phe180 & H-bond & 2.01 \\
\hline & & Ser181 & H-bond & 2.02 \\
\hline & & Asp273 & H-bond(side chain & 1.93 \\
\hline & & Phe277 & $\Pi-\Pi$ stacking & 5.06 \\
\hline \multirow{4}{*}{$2 \mathrm{~L}$} & \multirow{4}{*}{-25.95} & $\operatorname{Trp} 91$ & П-П stacking & 4.96 \\
\hline & & Phe180 & H-bond & 2.17 \\
\hline & & Ser181 & H-bond & 2.08 \\
\hline & & Ile254 & H-bond & 1.69 \\
\hline
\end{tabular}


The binding crevice for nicotinic acid was localized in the binding pocket (site) with trans-membrane helices 5 and 6 . The docking result of Nicotinic acid gives binding energy of $-17.68 \mathrm{Kcal} / \mathrm{mol}$. The oxygen atom (acidic group) of nicotinic acid forms hydrogen bonds with the Hydrogen atom of Phe180 at TMH5 and Trp256 at TMH6 having a bond distance of $1.89 \AA$ and $1.83 \AA$ respectively. The oxygen atom of Ile254 forms aromatic hydrogen bond with the hydrogen atom of Nicotinic acid at TMH6 and nicotinic acid also forms two ח-п interactions with Phe180 at TMH5 and Trp256 at TMH6 having a bond distance of $5.08 \AA$ and $4.32 \AA$ respectively (Figure 5).

Table 3. 2-Dimensional structures of nicotinic acid and its screened derivatives.

$\begin{gathered}\text { Compound } \\ \text { Name }\end{gathered}$
Nicotinic
Acid

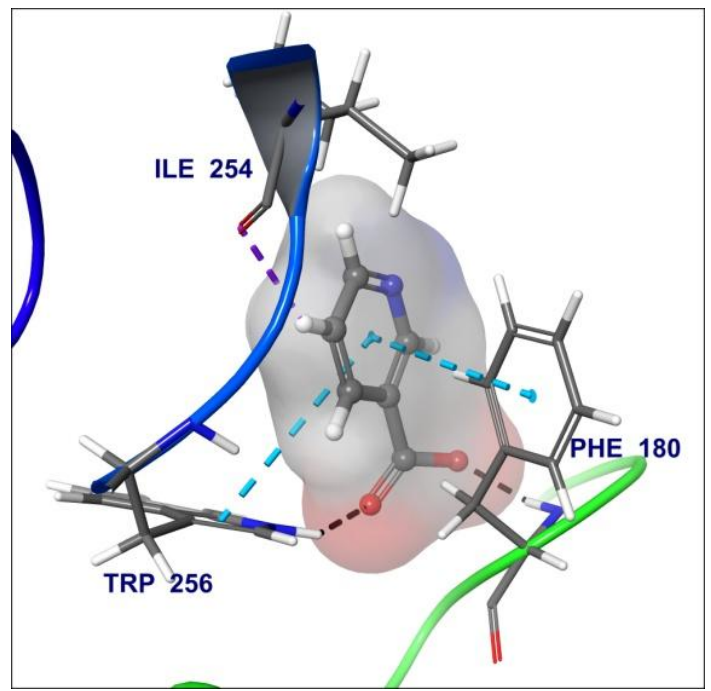

Figure 5. Nicotinic acid with its binding interactions in the binding pocket of the GPR109A receptor. The figure shows the docked pose of nicotinic acid within the binding pocket. The hydrogen bonds are represented by black dotted lines, $\Pi-\Pi$ interactions with blue dotted lines and aromatic hydrogen bond interactions with purple dotted lines. 
Out of 80 compounds, 78 compounds were found to have binding energy less than that of Nicotinic acid and only two compounds showed binding energy more than that of nicotinic acid i.e. compound $1 \mathrm{P}(\mathrm{N}-$ (thiazol-5-yl)-1H-1,2,4-triazole-3-carboxamide) and 1C ((S)-methyl 3-mercapto-2- (1H-1,2,4-triazole-3carboxamido) propionate) were having a binding energy of $-17.0198 \mathrm{kcal} / \mathrm{mol}$ and $-16.8273 \mathrm{kcal} / \mathrm{mol}$ respectively. All these ligands showed interactions with the GPCR109A which is similar to the interaction seen in nicotinic acid. It was found that the compounds 2B [(R)-methyl 2-(2-(1H-indol-3-yl) acetamido)-3(1H-indol-3-yl) propionate and 5J [N-(2H-benzo[d] [1, 2, 3] triazol-2-yl)-1H-pyrrole-2-carboxamide] having binding energy $-30.54 \mathrm{kcal} / \mathrm{mol}$ and $-27.17 \mathrm{kcal} / \mathrm{mol}$ respectively shows best binding interactions with GPR109A receptor. The binding crevice for $\mathrm{N}$ containing heterocyclic acids was localized in the binding pocket (site) with transmembrane helices 5, 6 and 7 and also ECL2 was critically involved in the ligand binding.

New lead compound 2B forms hydrogen bonds with Phe180, Asp273, and Ser181 with a bond distance of $1.87 \AA, 2.08 \AA$, and $2.18 \AA$. The Oxygen atom (ester Group) of the indole ring of compound $2 \mathrm{~B}$ was bound to Phe180 and Ser181 at TMH5 via H-bond, whereas indole ring nitrogen was simultaneously bound to Asp273 at TMH7 via aromatic H- bond. The indole ring also forms $\Pi-\Pi$ interactions with Phe276 at TMH7 and Phe180 at TMH5 (Figure 6(a)).

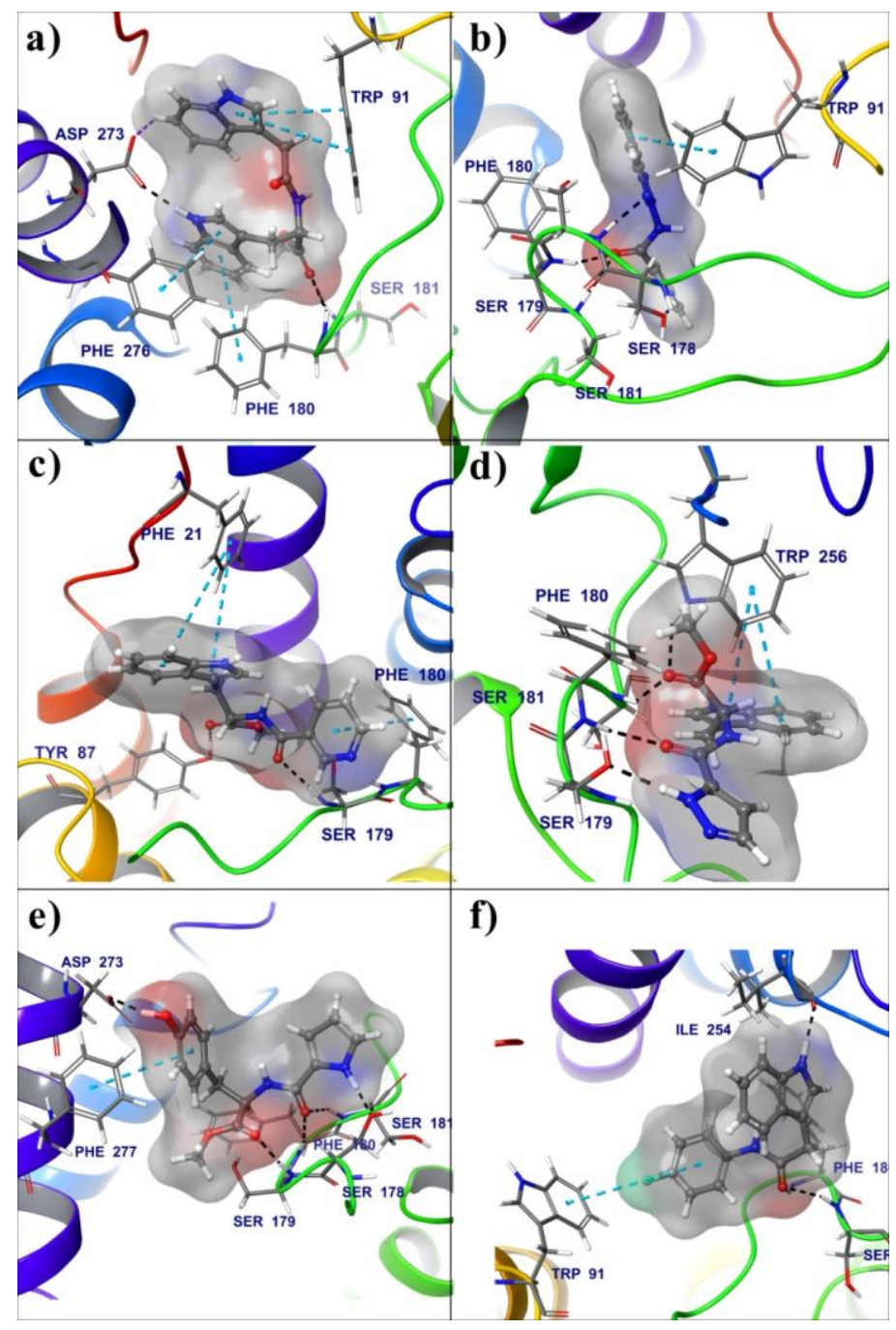

Figure 6. a) Docked pose of Compound 2B in the binding pocket of the GPCR109A receptor. (b) Compound $5 \mathrm{~J}$ in the binding pocket of the GPCR109A receptor. (c) Compound 3B in the binding pocket of theGPCR109A receptor. (d) Compound 4B in the binding pocket of the GPCR109A receptor. (e) Compound 5E in the binding pocket of the GPCR109A receptor. (f) Compound 2L in the binding pocket of the GPCR109A receptor. The figure shows the docking of the ligand within the active site. The GPR109A shown a ribbon diagram and all ligands are represented as ball and stick models. The hydrogen bonds are represented by black dotted lines, $\Pi-\Pi$ interactions with blue dotted lines and aromatic hydrogen bond interactions with purple dotted lines. 
Figure 6(b) shows that Compound 5J forms hydrogen bonds with Phe180, Ser179, Ser181, and Ser178 with the bond distance of $2.08 \AA, 1.95 \AA, 2.16 \AA$, and $1.59 \AA$. In compound $5 \mathrm{~J}$, an oxygen atom (ester Group) of pyrrole was bound to Phe180 and Ser181 at TMH5 via H-Bond. The nitrogen atom of the pyrrole ring forms a hydrogen bond with Ser178 at ECL2. The nitrogen of the triazole ring bound to Ser179 at ECL2 via H-bond.

In Figure 6(c) Compound 3B forms a hydrogen bond with Ser179 and Tyr87 having a bond distance of $2.05 \AA$ and $2.49 \AA$ respectively. In compound 3B, an oxygen atom (ester group) of pyridine ring and indole ring was bound to Ser179 at ECL2 and Tyr87 at TMH2 via H-Bond. Pyridine ring and indole ring form $\Pi-\Pi$ bonding with Phe180 at TMH5 and indole ring forms ח-п bonding Phe21 at TMH1.

Compound 4B forms H bond with Ser181, Trp256, Ser179, Phe180 having a bond distance of $1.68 \AA$, $2.11 \AA$, $1.99 \AA 2.44 \AA$ respectively. In Compound $4 \mathrm{~B}$, an oxygen atom (ester group) of the indole ring was bound to Ser181 at TMH5 and Trp256 at TMH7 via H bond, whereas oxygen atom of indole ring was bound to Phe180 via H-Bond. The nitrogen atom of the pyrazole ring forms a hydrogen bond with Ser179 at ECL2. The indole ring also forms $\Pi-\Pi$ interactions with Trp256 at TMH6 (Fig 6(d)).

Figure 6(e) shows that Compound 5E forms H bond with Ser181, Phe180, Ser179, Ser178 and Asp273 having a bond distance of $2.02 \AA, 2.01 \AA, 1.97 \AA, 1.55 \AA$ and $1.93 \AA$ respectively. In compound $5 \mathrm{E}$, an oxygen atom (ester group) of the Pyrrole ring was bound to Ser181 and Phe180 at TMH5 via H-bond whereas an acidic group of phenyl ring was bound to Ser179 at ECL2 via H-bond. The nitrogen atom of the pyrrole ring was bound to Ser178 at ECL2 via H-Bond. Whereas the OH group of tyrosine forms H-bond with Asp273 at TMH7. Phe277 was embedded in phenyl ring via ח-п stacking.

In Figure 6(f), Compound 2L forms H-bond with Phe180, Ser181 and Ile254 having the bond distance of $2.08 \AA, 2.17 \AA$ and $1.69 \AA$ respectively. In compound $2 \mathrm{~L}$ oxygen atom (ester group) of the indole ring forms a hydrogen bond with Phe180 and Ser181 at TMH5. Whereas oxygen atom of Ile254 at TMH6 forms a hydrogen bond with the nitrogen atom of the indole ring. Trp91 was embedded in phenyl ring via $\Pi-\Pi$ bonding.

\subsection{Simulation interaction analysis}

The thermodynamic stability of the complex system i.e. GPCR109A-2B [(R)-methyl 2-(2-(1H-indol-3yl) acetamido)-3-(1H-indol-3-yl) was examined using the parameters RMSD (Root Mean Square Deviation) (Figure 7) and RMSFs (Root Means Square Fluctuations) (Figure 8) using 1000 trajectory captured during molecular dynamic simulation [28]. For this simulation, a 100ns unconstrained simulation was performed on the docked complex structure of GPCR109A bound to best docked pose of compound 2B. Despite the initial structural arrangements of the docked complex, the average RMSD of the trajectories for bound protein backbone atoms showed relative stability. Figure 7 shows, the stable RMSD values of the atoms for docked compound 2B with the GPCR109A receptor. The RMSD analysis for GPCR109A indicates that they reach equilibration and oscillate around an average value after 40ns. The average RMSDs from 40ns to 100ns for compound 2B bound to GPCR109A receptor was $3.0 \AA$.

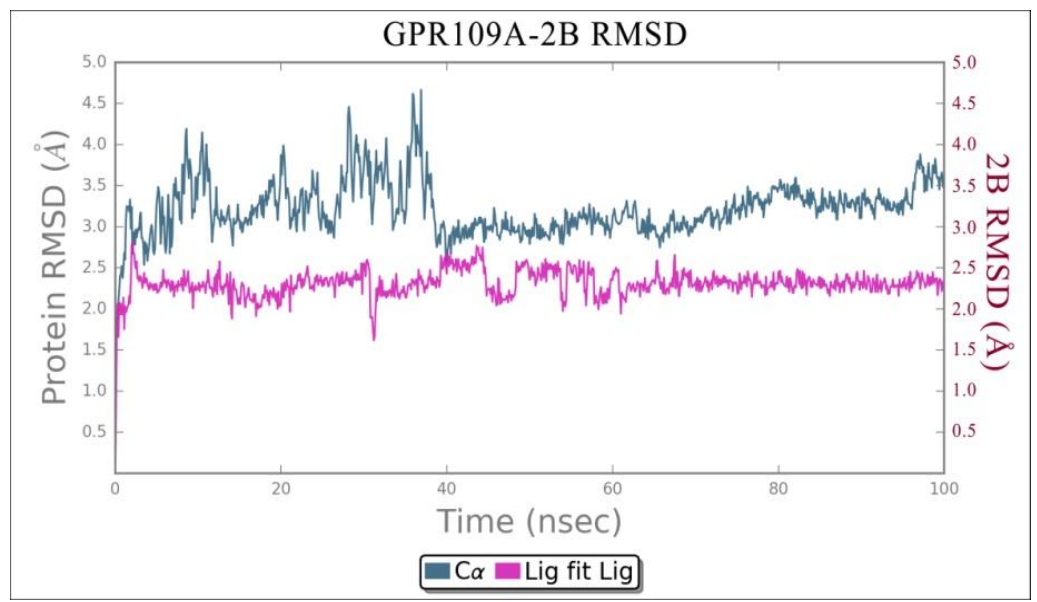

Figure 7. Root mean square deviations (RMSD) of GPR109A and lead compound 2B, during 100ns MD simulation. 


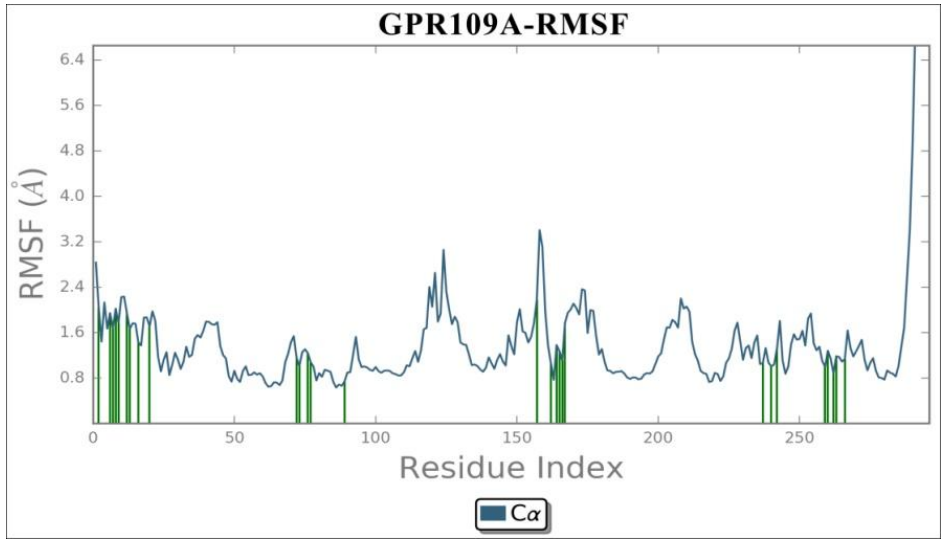

Figure 8. Root mean square fluctuation (RMSF) of the GPR109A model upon binding of lead compound 2B.

These RMSD results showed the relative stability of compound 2B bound to the GPCR109A receptor throughout the simulation. This result suggests that compound $2 \mathrm{~B}$ undergoes a minimal structural conformational change during the 100ns simulations. Local protein quality was analyzed by measuring the time-averaged RMSF value of compound 2B after binding with the GPCR109A receptor against residue numbers based on 100ns trajectory data. The average RMSFs measured for compound 2B (Figure 8) bound to GPR109A was 1.7 $\AA$, which reveals the relative stability of compound 2B upon binding with GPCR109A. The protein-ligand interaction diagram during 100ns simulation for compound 2B bound to GPCR109A receptor, suggests that designed lead compound $2 \mathrm{~B}$ shows a more binding pattern of hydrogen bonding, ionic interaction, hydrophobic interaction as well as water bridges.

\section{CONCLUSION}

The present study focuses on the novel nitrogen heterocycles as GPR109A agonists as potential antihyperlipidemic agents. The protein 3D structure was built by using homology modeling. Then the model structure was refined by the energy minimization molecular dynamics methods. In our studies, the residues Ser181, Ser178, Phe180, Ser179, Phe276, Leu176, Trp91, Phe21, Val272, His259, Asp273, Tyr269, Ile254, and Thr260 are important for strong hydrogen bonding interaction with the substrate. From the molecular docking study, it was observed that among 80 ligands screened according to their binding energy, interaction pattern and their bond distances, it is found that new lead compound $2 \mathrm{~B}$ is having more affinity compared to natural agonist nicotinic acid. From this study, it was clear that the interaction of Phe180 and Trp256 residues are more favorable to achieve stable conformation of compound $2 \mathrm{~B}$ into the binding cavity of the GPR109A receptor. The graph of the binding pattern generated from the MD simulation study shows specific interactions with the reference compound nicotinic acid.

Our study reveals that new lead compound $2 \mathrm{~B}$ having more binding affinity, structural stability and is also favorable dynamically than other known compounds. From the above docking results, it can be concluded that the ester group of nitrogen-containing heterocyclic rings shows more interactions with the GPR109A receptor than an acidic group of pyridine ring which was present in nicotinic acid. The data combined with the model also suggests the possibility that some aromatic residues, such as Phe180, and Trp256, Ser179, Ser181plays an important role in the formation of a gateway that allows $\mathrm{N}$ containing heterocyclic acids to access the binding pocket. The current study helps in shedding light on the antihyperlipidemic effects of nicotinic acid analogs. Data obtained from the study will serve as the reference for in vivo and in vitro studies.

\section{MATERIALS AND METHODS}

\subsection{Homology modeling}

\subsubsection{Template selection for GPR109A}

The sequence of GPR109A was retrieved from UniProt [29] database (Uniprot ID: Q8TDS4) searched against Protein Data Bank using the BLAST-p server [30] with specifically to Homo sapiens (Taxid: 9606). As a result, the X-ray crystal structure of the human P2Y1 receptor (PDB ID: 4XNW), with $2.7 \AA$ resolution share 
$28.52 \%$ identity with the sequence of GPR109A and it was considered as a template [31] for further studies. The pairwise sequence alignment was done for the sequence of GPR109A and template 4XNW using Maestro.

\subsubsection{D structure building of GPR109A and its validation}

SWISS-MODEL server [19] was used to generate homology models of GPR109A by taking backbone C-a atom information of the Human P2Y1 receptor crystal structure. The chain A of the template 4XNW was considered for homology modeling, as it shares the high sequence similarity with the GPR109A. Generated 3D model structure of GPR109A was retrieved from the SWISS-MODEL server and used for energy minimization to obtain the stable confirmation. Further, the GPR109A model was then subjected to stereochemical validation using PROCHECK [20], PROSA Web server [21] and SAVES [22] were employed to select a high-quality model for docking studies. Ramachandran plot was generated for the model to visualize energetically allowed regions for backbone $\psi$ (psi) and $\varphi$ (phi) dihedral angles of amino acid residues using Maestro [25]. From the stereochemical validation study, the valid model of GPR109A was selected for further calculations.

\subsubsection{Preparation of GPR109A Model and Ligands}

Protein Preparation Wizard (PPW) [32] was used to refine the valid GPR109A model by applying the OPLS-2005 force field [26] with a root mean square deviation (RMSD) tolerance on C-a atom of GPR109A model $0.3 \AA$. The protonation and ionization state of basic and acidic groups amino acids were chosen using the PROPKA program [25] according to physiological $\mathrm{pH}$ 7.4. The 2D structures of nicotinic acid, 1, 2, 4triazole carboxylic acid, pyrrole-2-carboxylic acid, indole-3-acetic acid, pyrazole-3-carboxylic acid, and its derivatives were drawn on Marvin Sketch software [33]and visualized with Maestro. All the ligands were energy minimized using the OPLS-2005 force field in Maestro to get energetically favorable conformation.

\subsubsection{Molecular docking calculations}

To reveal the binding pattern of selected compounds and their derivatives within the binding cavity of the GPR109A model, we performed the molecular docking calculations. The binding cavity for the GPR109A model was mapped considering earlier reported amino acid residues [13] Ser181, Ser178, Phe180, Ser179, Phe276, Leu176, Trp91, Phe21, Val272, His259, Asp273, Tyr269, Ile254, and Thr260. The cavity of the GPR109A was sufficient for ligand conformations and calculations. All ligands were subjected to molecular docking calculations with the GPR109A model by using FlexX software [27]. All atoms from the GPR109A model are treated as rigid during docking calculations. FlexX uses incremental construction algorithm [34]. The docking parameters are kept at its default value of software and numbers of possible conformation for each derivative were generated. Based on docking score and ligand interaction analysis with the GPR109A receptor, the best poses for a given ligand having greater binding affinity toward GPR109A was selected as lead compounds.

\subsubsection{Molecular dynamic simulation}

Molecular Dynamic (MD) simulations were performed for the complex of GPR109A with lead molecule $2 \mathrm{~B}$ having greater binding affinity $(-30.54 \mathrm{kcal} / \mathrm{mol})$. To determine the binding strength of ligands within the GPR109A binding pocket after docking calculation, MD simulation was run up to 100ns time using Desmond [28]. This process is useful to calculate forces, compute the motion of atoms. However, Desmond incorporates a more detailed temperature, pressure, volume system and has more functionality built-in for executing protein-ligand interactions. Using the system builder of Desmond in the Maestro program [35], the system for both complexes was immersed in the water-filled cubic box containing 7149 water molecules using an extended simple point charge (SPC), a three-point water model within periodic boundary conditions. The total charge of the solvent system is neutralized by adding 16 chlorine ions (Cl-) to the complex systems. Energy minimization is a very essential step in MD and it is done using the steepest descent method. Cubic box type (with box size $10 \AA$ ) is considered for minimizing edge effects in a finite system to apply periodic boundary conditions. The atoms of the system to be simulated are put into the space-filling box, which is surrounded by translated copies of itself. OPLS-2005 force field (parameters used to describe the potential energy of a system) is chosen in this work which is an improved force field suited for molecular dynamics simulations of proteins. 
Acknowledgment: Authors are thankful to Dr. D. Y. Patil Biotechnology and Bioinformatics Institute, Dr. D. Y. Patil Vidyapeeth, Pune, and Dr. D. Y. Patil Institute of Pharmaceutical Sciences and Research, for the physical infrastructure and working space. Authors acknowledge the support of the Department of Science and TechnologyScience and Engineering Research Board (DST-SERB), Govt. of India, New Delhi under the Young Scientist Scheme (YSS/2015/002035), for providing a super optimized computer to perform docking and dynamics calculations. Senior Research Fellowship awarded to Mr. Kiran Bharat Lokhande (Project ID: 2019-3458; File No.: ISRM/11(54)/2019) of Indian Council of Medical Research, New Delhi is also acknowledged.

Author contributions: Concept - A.T., P.K., K.L.; Design - K.L., P.K., A.T.; Supervision - A.T., K.S., P.K.; Resources A.T., P.K., S.C., K.S.; Materials - A.T., K.S., S.C.; Data Collection and/or Processing - P.K., K.L., A.T.; Analysis and/or Interpretation - P.K., K.L., A.T., K.S.; Literature Search - P.K., A.T., K.L, K.S.; Writing - K.L., P.K., A.T., K.S., SC.; Critical Reviews - P.K., A.T., K.L., K.S., S.C.

Conflict of interest statement: The authors declared no conflict of interest in the manuscript.

\section{REFERENCES}

[1] Altschul R, Hoffer A, Stephen JD. Influence of nicotinic acid on serum cholesterol in man. Arch Biochem. 1995; 54(2): 558-559. [CrossRef]

[2] Carlson LA, Oro L. The effect of nicotinic acid on the plasma free fatty acid; demonstration of a metabolic type of sympa-thicolysis. Acta Med Scand. 1962; 172: 641-645. [CrossRef]

[3] Blum CB, Levy RI, Eisenberg S, Hall M, Goebel RH, Berman M. High density lipoprotein metabolism in man. J Clin Invest. 1977; 60(4): 795-807. [CrossRef]

[4] Olsson AG. Principles and Treatment of Lipoprotein Disorders. Handbook of Experi-mental Pharmacology. 1994; 109: 349-400. [CrossRef]

[5] Aktories K, Schultz G, Jakobs KH, , Regulation of adenyl atecyclase activity in hamster adipocytes, Inhibition by prostaglandins. alpha-adrenergic agonists and nicotinic acid. Naunyn-Schmiedeberg's Arch Pharmacol. 1980; 312: 167- 173. [CrossRef]

[6] Aktories K, Schultz G, Jakobs KH. Inactivation of the guanine nucleotide regulatory site mediating inhibition of the adeny-late cyclase in hamster adipocytes. Naunyn-Schmiedeberg's Arch Pharmacol. 1982; 321(4): 247-252.

[7] Tornvall P, Hamsten A, Johansson J, Carlson LA. Normalisation of the composition of very low density lipoprotein in hypertriglyceridemia by nicotinic acid. Atherosclerosis. 1990; 84(2-3): 219-227. [CrossRef]

[8] Soga T, Kamohara M, Takasaki J, Matsumoto S, Saito T, Ohishi T, et al. Molecular identification of nicotinic acid receptor. Biochem Biophys Res Commun. 2003; 303(1): 364-369. [CrossRef]

[9] Tunaru S, Kero J, Schaub A, Wufka C, Blaukat A, Pfeffer K, et al. PUMA-G and HM74 are receptors for nicotinic acid and mediate its antilipolytic effect. Nat Med. 2003; 9(3): 352-355. [CrossRef]

[10] Wise A, Foord SM, Fraser NJ, Barnes AA, Elshourbagy N, Eilert M, et al. Molecular identification of high and low affinity receptors for nicotinic acid. J Biol Chem. 2003; 278(11): 9869-9874. [CrossRef]

[11] Lee DK, Nguyen T, Lynch KR, Cheng R, Vanti WB, Arkhitko O, et al. Discovery and mapping of ten novel G. Protein coupled receptor genes. Gene. 2001; 275(1): 83-91. [CrossRef]

[12] Teller DC, Okada T, Behnke CA, Palczewski K, Stenkamp RE, Advances in determination of a high-resolution three-dimensional structure of rhodopsin. A model of G-protein-coupled receptors (GPCRs). Biochem. 2001; 40(26): 7761-7772. [CrossRef]

[13] Tanura s, Jens L, Jukka K, Gerd K, Stefan O. Characterization of Determinants of Ligand Binding to the Nicotinic Acid Receptor GPR109A (HM74A/PUMA-G). Mol Pharmacol 2005; 68(5): 1271-1280. [CrossRef]

[14] Berman HM, Westbrook J, Feng Z, Gilliland G, Bhat TN, Weissig H, et al. The Protein Data Bank. Nucleic Acids Res. 2000; 28(1): 235-242. [CrossRef]

[15] Laskowski RA, Rullmannn JA, MacArthur MW, Kaptein R, Thornton JM. AQUA and PROCHECK-NMR: programs for checking the quality of protein structures solved by NMR. J Biomol NMR. 1996; 8(4): 477-486. [CrossRef]

[16] Shepherd J, Packard CJ, Patsch JR, Gotto AM, Taunton OD. Effects of nicotinic acid therapy on plasma high density lipoprotein subfraction distribution and composition and on apolipoprotein A metabolism. J Clin Invest. 1979; 63(5): 858- 867. [CrossRef] 
[17] Goldsmith GA, Cordill S. The vasodilating effects of nicotinic acid (relation to metabolic rate and body temperature). Am J Med Sci. 1943; 205: 204-208.

[18] Benyo Z, Gille A, Kero J, Csiky M, Suchánková MC, Nüsing RM, et al. GPR109A (HM74A/PUMA-G) mediates nicotinic acid induced flushing. J Clin Invest. 2005; 115(12): 3634-3640. [CrossRef]

[19] Waterhouse A, Bertoni M, Bienert S, Studer G, Tauriello G, Gumienny R, et al. SWISS-MODEL: homology modelling of protein structures and complexes. Nucleic Acids Res. 2018; 46 (W1): W296-W303. [CrossRef]

[20] Laskowski RA, MacArthur MW, Moss DS, Thornton JM. PROCHECK - a program to check the stereochemical quality of protein structures. J App Cryst. 1993; 26(2): 283-291. [CrossRef]

[21] Markus W, Sippl MJ. ProSA-web: interactive web service for the recognition of errors in three-dimensional structures of proteins. Nucleic Acids Res. 2007; 35: W407-W410. [CrossRef]

[22] Eisenberg D, Lüthy R, Bowie JU. VERIFY3D: assessment of protein models with three-dimensional profiles. Methods Enzymol. 1997; 277: 396-404. [CrossRef]

[23] Roman AL. PDBsum new things. Nucleic Acids Res. 2009; 37(1): D355-D359. [CrossRef]

[24] Pandy-Szekeres G, Munk C, Tsonkov TM, Mordalski S, Harpsøe K, Hauser AS, Bojarski AJ. Gloriam DE. GPCRdb in 2018: adding GPCR structure models and ligands. Nucleic Acids Res. 2018; 46 (D1): D440-D448. [CrossRef]

[25] Schrodinger Release 2018-1: Maestro. Schrodinger, LLC, New York, NY, 2018.

[26] DuBay KH, Hall ML, Hughes TF, Wu C, Reichman DR, Friesner RA. Accurate Force Field Development for Modeling Conjugated Polymers. J Chem Theory Comput. 2012; 8(11): 4556-4569. [CrossRef]

[27] Rarey M, Kramer B, Lengauer T, Klebe G. A fast flexible docking method using an incremental construction algorithm. J Mol Biol. 1996; 261(3): 470-489. [CrossRef]

[28] Kevin J, Bowers C Edmond, Huafeng X, Ron OD, Michael PE, Brent AG, et al. . Scalable Algorithms for Molecular Dynamics Simulations on Commodity Clusters. Proceedings of the ACM/IEEE Conference on Supercomputing (SC06) 2006. [CrossRef]

[29] Chen C, Huang H, Wu CH. Protein Bioinformatics Databases and Resources Methods. Mol Biol. 2017;1558: 3-39. [CrossRef]

[30] McGinnis S, Madden TL. BLAST: at the core of a powerful and diverse set of sequence analysis tools. Nucleic Acids Res. 2004; 32:W20-W25. [CrossRef]

[31] Zhang D, Gao ZG, Zhang K, Kiselev E, Crane S, Wang J, et al. Two disparate ligand-binding sites in the human P2Y1 receptor. Nature. 2015; 520(7547): 317-321. [CrossRef]

[32] Schrodinger Release 2018-1: Protein Preparation Wizard. Schrodinger, LLC, New York, NY, 2018.

[33] ChemAxon L., Marvin Sketch 5114 (2012).

[34] Badry DB, Maxim T, Ruben A, Charles LB. Comparative study of several algorithms for flexible ligand docking. J Comput Aid Mol Des. 2003; 17(11): 755-763. [CrossRef]

[35] Schrodinger Release 2017-4: Desmond Molecular Dynamics System, D. E. Shaw Research, New York, NY. MaestroDesmond Interoperability Tools. Schrodinger, LLC, New York, NY, 2017.

This is an open access article which is publicly available on our journal's website under Institutional Repository at http://dspace.marmara.edu.tr. 